Mots. Les langages du politique

Guerres et paix. Débats, combats, polémiques

Presse écrite et traitement immédiat du 11 septembre : un imaginaire occidental réactivé ?

Frédéric Ramel

\title{
OpenEdition
}

Journals

Édition électronique

URL : https://journals.openedition.org/mots/2653

DOI : $10.4000 /$ mots. 2653

ISSN : 1960-6001

Éditeur

ENS Éditions

Édition imprimée

Date de publication : 1 novembre 2004

Pagination : 113-126

ISBN : 2-84788-064-X

ISSN : 0243-6450

Référence électronique

Frédéric Ramel, «Presse écrite et traitement immédiat du 11 septembre : un imaginaire occidental réactivé ? ", Mots. Les langages du politique [En ligne], 76 | 2004, mis en ligne le 21 avril 2008, consulté le 23 avril 2022. URL : http://journals.openedition.org/mots/2653; DOl : https://doi.org/10.4000/ mots.2653 


\section{Presse écrite et traitement immédiat du 11 septembre : un imaginaire occidental réactivé ?}

Durant la guerre froide, le prisme par lequel s'élaborait la désignation de l'ennemi soviétique a reposé sur la stigmatisation de la logique totalitaire et militariste. Cette condamnation sans appel du système politique se manifestait tout particulièrement lors de crises internationales. La destruction d'un Boeing sud-coréen en 1983 s'inscrivait par exemple dans cette perspective simplificatrice qui oriente l'interprétation de l'évènement en fonction de l'idéologie dominante $^{2}$. Les discours politiques des gouvernants ne constituent pas le seul matériau pertinent à cet égard. La presse écrite également offre une modalité d'accès significative aux images réductionnistes ${ }^{3}$ de l'ennemi. Elle permet de saisir des mutations dans les représentations stratégiques, notamment dans l'après-guerre froide. Ainsi, l'un des traits saillants depuis la guerre du Golfe en 1991 réside dans une substitution tendancielle de l'ennemi «vert» au sud à l'ennemi rouge soviétique de l'Est ${ }^{4}$ ce qui tend à renforcer la légitimité de certains paradigmes critiquables, tel le choc des civilisations ${ }^{5}$.

Les évènements du 11 septembre semblent avoir révélé à nouveau ce mécanisme de transfert. Ils créent un contexte de communication favorable à la réactivation de préjugés occidentaux quant à l'altérité arabo-musulmane ${ }^{6}$. Après une

1. Centre lyonnais d'études sur la sécurité internationale et la défense - Université Jean Moulin, Lyon 3 - framel@free.fr

2. Il s'inscrit dans une vaste campagne américaine visant à déceler le potentiel exact de la défense anti-aérienne le long des frontières soviétiques. Il s'agit d'une opération de sondage passif afin de déclencher les radars de la région «Extrême-Orient soviétique stratégique». A. Ilyashov, 1994, «Le réexamen des affaires du vol 007 de la Korean Airlines et du R-B 50 », Études internationales, vol. XXV, $\mathrm{n}^{\circ} 1, \mathrm{p} .4764$.

3. R. Ottosen, 1995, «Enemy Images and the Journalistic Process», Journal of Peace Research, vol. $32, \mathrm{n}^{\circ} 1$, p. 97-112.

4. R. El Kareth, 1991, «Les médias français et la crise du Golfe», Revue d'études palestiniennes, $\mathrm{n}^{\circ} 38$, hiver, p. 63-74.

5. S. Huntington, 1997, Le choc des civilisations, Paris, Odile Jacob.

6. La formation d'une identité (individuelle ou collective) repose sur des effets en miroir 
courte généalogie des stéréotypes occidentaux à l'encontre de l'islam et de l'arabité, on procédera à une étude comparative de la presse écrite française durant le mois de septembre 2001 jusqu'au déclenchement de l'attaque américaine en Afghanistan (du 12 septembre au 8 octobre). Le corpus se compose des trois principaux quotidiens nationaux (Le Monde, Le Figaro, Libération) et de trois hebdomadaires grand public (Le Point, Le Nouvel Observateur, L'Express) : on entend ainsi repérer une orientation argumentative ${ }^{7}$ commune au-delà d'une hétérogénéité de vecteur médiatique ${ }^{8}$. Une telle orientation tend à vérifier l'hypothèse selon laquelle la désignation de l'ennemi «al-Qaida» se sédimente autour d'un imaginaire occidental qui, sans répondre de manière exhaustive aux prédicats culturalistes de Huntington, repose sur des procédés d'amnésie et d'occultations 9 .

Étudier les mécanismes sous-jacents à l'énonciation médiatique n'est pas chose aisée, tout particulièrement lorsque la réflexion porte sur la formulation de stéréotypes ou de préjugés culturels ${ }^{10}$. L'étude est susceptible de se heurter aux intérêts économiques ainsi qu'au prestige des organes médiatiques. L'analyse doit reposer sur une méthodologie particulièrement transparente. La presse est une voie propice pour déceler des représentations. Un journal véhicule un imaginaire social dont le récepteur est censé partager les prédicats ${ }^{11}$. Dans la situation d'après-guerre froide, la substitution d'images de l'ennemi donne une nouvelle actualité aux représentations imaginaires de l'altérité arabo-musulmane et, plus largement, à des conceptions dichotomiques entre l'Orient et

(différenciation, reconnaissance). J. Lacan, 1966, Écrits I, Paris, Seuil, p. 90 et suiv. La conscience européenne et occidentale s'est, quant à elle, édifiée largement par rapport à l'altérité arabo-musulmane.

7. Voir S. Bonnafous, 1996, «La gestion de l'incertain par les médias contemporains dans la crise yougoslave», Mots, $\mathrm{n}^{\circ} 47$, juin, p. 11.

8. Ce qui permet de souligner l'existence d'une «exposition discursive» commune des citoyens par rapport à l'interprétation d'un évènement. Sur ce concept d'exposition discursive, voir C. Develotte, 1994, «Espace professionnel, espace discursif dans l'institution éducative», Parcours linguistique de discours spécialisés, Berne, Peter Lang, p. 133-144.

9. H. Maler, 2002, «Médias en guerre», Revue d'études palestiniennes, n 82, p. 28-33.

10. C. Lavergne et P. Siblot, 1993, «Les fabriques de sens commun : presse régionale et discours d'exclusion», Hommes et migrations, octobre, p. 34-37. Les deux linguistes montpelliérains suscitèrent une controverse juridique en analysant une récurrence significative au sein de la presse régionale : les immigrés y sont étroitement associés soit à la délinquance, soit au chômage (une thématique xénophobe courante qui assimile l'étranger à un fauteur de troubles menaçant deux droits fondamentaux : le travail et la sécurité publique).

11. P. Laborie, 1988, «De l'opinion publique à l'imaginaire social», $X X^{e}$ siècle, avril-juin, p. 101-117; P. Laborie, 1990, L'opinion publique sous Vichy, Paris, Seuil, p. 11-55. Les travaux de Laborie constituent ici un tremplin méthodologique fécond par rapport à l'objet d'analyse. 
l'Occident. Afin de préciser la nature de ces représentations, il convient d'en dégager les matrices historiques et mnésiques susceptibles de trouver dans la presse écrite un vecteur d'expression.

Parmi les différents objectifs que se fixe toute étude de l'opinion publique sous l'angle de la presse, Pierre Laborie distingue les modalités de construction des représentations et l'influence de l'imaginaire collectif ${ }^{12}$. Le premier aspect renvoie à la morphologie des représentations, lesquelles globalisent, déforment et réduisent. Ces procédés de schématisation du réel reposent largement sur des perceptions dichotomiques de l'évènement dont le registre est le plus souvent de l'ordre du sensible et de l'affectif afin de susciter une résonance majeure auprès du récepteur : «on réduit le complexe au rudimentaire ${ }^{13}$. Le second aspect consiste à déceler à travers les représentations, des séquences de l'imaginaire social; c'est-à-dire des moments au cours desquels se manifeste la faculté de réactualiser des héritages. Ainsi, «les représentations sont le lieu où les perceptions instables de l'immédiat viennent converger et interférer à la fois avec les héritages de mémoire des longues durées et avec les fantasmes de projections divinatoires $\gg{ }^{14}$. Certains éléments du passé sont accentués ou bien au contraire occultés. S'opère par là un double mécanisme : celui de la réappropriation d'un héritage mnésique et/ou historique particulier et celui de l'oubli ou de l'amnésie. Ces pans de mémoire reprennent consistance dans le présent en livrant une interprétation des évènements pris sur le vif selon des perceptions ancestrales. Enfin, l'imaginaire anticipe le futur grâce au processus de projection ou bien d'autoprophétie réalisatrice.

Finalement, l'imaginaire produit du sens par rémanence ou occultation. Il favorise une lecture biaisée de la réalité politique et sociale. En ce qui concerne le contexte postérieur à la guerre froide, des représentations de l'ennemi au sud tendent à se développer selon les mécanismes décrits. Elles reposent sur une mythification de l'altérité arabo-musulmane, notamment l'idée d'une coupure radicale et immuable entre Orient et Occident.

12. P. Laborie souligne les facteurs situationnels. Étudier les représentations inhérentes aux énoncés médiatiques trouve dans les périodes de crises, ou de «mutations tumultueuses», des contextes favorables d'études. Cette perspective rejoint l'approche de G. Duby : les structures latentes ordinairement cachées que sont les représentations émergent alors avec plus d'intensité. G. Duby, 1974, «Histoires sociales et idéologies des sociétés» J. Le Goff et P. Nora (éd.), 1974, Faire de l'histoire, Paris, Gallimard (Folio), p. 216.

13. P. Laborie, ouvr. cité, p. 50.

14. P. Laborie, «De l'opinion publique à l'imaginaire social», ouvr. cité, p. 117. 


\section{Un imaginaire occidental mythifié}

Les matrices de l'imaginaire occidental ont un caractère mythique. Le terme mythe renvoie ici à deux acceptions fondamentales. D'une part, la genèse de la coupure Orient/Occident est située à des époques au cours desquelles elle n'a ni sens ni consistance (le mythe des origines). D'autre part, cette coupure permet de fixer l'image de l'Orient et de l'Occident indépendamment de toute évolution historique (le mythe d'ordre immuable hors du temps). Trois mythes constituent l'épine dorsale de cette dichotomie : la Grèce antique, l'apparition de l'islam au $7^{\mathrm{e}}$ siècle et les croisades.

Nombre d'auteurs ont soutenu que l'Orient fut perçu par l'Occident comme son grand contraire complémentaire dès la période hellénistique. Les guerres médiques opposant Perses et Grecs constitueraient le fond mythique initial de cet antagonisme. Sous la plume d'André Siegfried ${ }^{15}$ ou de Bernard Lewis ${ }^{16}$, cette conception se révèle largement anachronique dans la mesure où les penseurs grecs ne raisonnaient pas en ces termes, la Grèce étant considérée comme la charnière des deux mondes ${ }^{17}$. Le second mythe sur lequel l'imaginaire occidental se fonde réside dans l'émergence de l'islam. L'apparition d'une nouvelle religion au sein de l'espace méditerranéen est censée constituer l'un des points nodaux de la coupure Orient/Occident. Cet aspect est largement popularisé sous la plume d'Henri Pirenne ou de Bernard Lewis, selon lesquels deux civilisations «hostiles ${ }^{18}{ }^{8}$ ' affrontent dont l'une s'appuie sur la guerre sainte afin de rayonner ${ }^{19}$. Cette façon de raisonner fait fi de la réalité perçue à l'époque car, comme le souligne Thierry Hentsch, l'expansion arabe au $8^{\mathrm{e}}$ siècle ne semble pas constituer la préoccupation majeure de l'Occident chrétien. L'opposition

15. Voir la conception de l'un des pères de la science politique en France, dégagée dans T. Hentsch, 1988, L'Orient imaginaire. La vision politique occidentale de l'Est méditerranéen, Paris, Minuit, p. 26.

16. À partir des deux célèbres tragédies que sont Les Perses d'Eschyle et Les Bacchantes d'Euripide, il décèle une ligne de fracture entre un Occident rationnel et supérieur et un Orient excessif en proie aux débordements intempestifs. E. Saïd, 1980, L'orientalisme. L'Orient créé par l'Occident, Paris, Seuil, p. 73.

17. S. Amin, 1988, L'eurocentrisme. Critique d'une idéologie, Paris, Éditions AnthroposEconomica, p. 63.

18. H. Pirenne (éd.), 1987, Mahomet et Charlemagne, Milan, Jaca Book, p. 68.

19. C'est là l'hypothèse de Bernard Lewis qui soutient que la religion musulmane repose sur une loi de persistance du djihad en tant qu'affrontement avec les impies. B. Lewis, 1992, Europe/Islam. Actions réactions, Paris, Gallimard, p. 22. 
Orient/Occident sous la forme Islam/chrétienté n'existe pas encore ${ }^{20}$. C'est le schisme de Byzance qui inquiète le plus la chrétienté occidentale. Enfin, la dernière matrice mythique correspond aux croisades. L'image de l'hérétique produite et véhiculée par la papauté notamment à travers l'appel d'Urbain II au concile de Clermont en 1095 est ici le nerf du discours sur l'altérité arabo-musulmane. Il s'agit de libérer les Lieux saints tout en éradiquant une conception doctrinale considérée comme impie. Là encore, des distorsions surgissent avec la réalité historique dans le sens où les gens de Jérusalem n'éprouvent nul besoin d'être libérés. Les croisades répondent d'ailleurs plus à une congruence d'objectifs à la fois internes (enrayer les querelles frappant l'Europe chrétienne) et externes (détruire la puissance économique et politique de Byzance).

Cette construction mythique insérant l'ennemi arabe et musulman au sein d'une opposition immuable Orient/Occident s'articule autour de ces trois éléments saillants. Mais fondamentalement, c'est à partir de la Renaissance que l'imaginaire se formule avec une grande netteté. Occultant l'héritage andalou sur lequel l'Europe intellectuelle s'est pourtant épanouie via la rencontre avec Avicenne, Averroès et... Aristote, cette période historique apparait comme «une rupture décidée, profonde, consciente avec le monde philosophique et culturel musulman ${ }^{21}$. L'altérité arabo-musulmane est conçue comme une source de barbarie et d'impiété. Cette perception négative se renforce avec les conquêtes territoriales des Turcs puisque leur Empire est associé à la «terreur du monde». La prise de Constantinople en 1453 provoque un choc symbolique dans toute la chrétienté22. Face au péril, hommes d'Église et humanistes présentent les succès rencontrés par l'Empire ottoman comme autant de sanctions divines à l'encontre des chrétiens ${ }^{23}$.

Ces spécificités sont-elles réactivées dans la période de l'après-guerre froide? La fin de la bipolarité entraine une évanescence de la logique totalitaire à partir de laquelle les conflits et les crises pouvaient faire l'objet d'une intelligibilité pendant la guerre froide. Le basculement dans un autre monde stimule-t-il la mobilisation d'autres manières d'appréhender la réalité stratégique et notamment, à reformuler cet imaginaire occidental quant à l'altérité arabo-musulmane ? L'étude des médias pendant la guerre du Golfe révèle toute la puissance d'un tel imaginaire et permet de répondre par l'affirmative à cette interrogation.

20. T. Hentsch, ouvr. cité, p. 41.

21. A. de Libéra, 1992, «Comment l'Europe a découvert l'Islam» dans Connaissance de l'Islam, Paris, Syros, p. 60.

22. J. Delumeau, 1978, La peur en Occident, Paris, Fayard, p. 263.

23. C'est le cas d'Érasme et de Luther, ibid., p. 270-271. 
Cependant, est-il également à l'œuvre après le 11 septembre ? Comment se donne-til lors de cette crise ? Se caractérise-til par une certaine isomorphie?

\section{Décrire et expliquer le 11 septembre : une reformulation de l'imaginaire occidental}

Entre les attentats du 11 septembre et la mise en place de l'opération militaire organisée par les États-Unis en Afghanistan au début d'octobre 2001, la presse écrite française livre ses premières interprétations de l'évènement. Trois propriétés communes caractérisent le traitement de l'information durant cette période : les journaux soulignent l'intensité des attentats et la rupture stratégique qu'ils incarnent dans l'histoire de l'après-guerre froide; la perception d'un tournant fondamental au sein du système international contemporain conditionne la réactivation de l'imaginaire occidental mais sous une forme poreuse; ce qui favorise l'expression d'un imaginaire toujours actif mais perceptible à travers des silences ainsi que des occultations dans la façon d'expliquer l'inimaginable.

\section{Un imaginaire mobilisé par la nature historique de l'évènement}

Toutes les parutions médiatiques à l'issue du 11 septembre insistent sur deux traits majeurs quant aux attentats : leur intensité et leur nature historique. L'intensité se manifeste du point de vue iconographique et photographique ${ }^{24}$ mais aussi et surtout à travers les vecteurs linguistiques. Les titres choisis, lesquels condensent l'information et orientent la première interprétation de l'évènement par le lecteur ${ }^{25}$, révèlent une dimension conflictuelle quasi paroxystique des attentats. À maintes reprises, le mot de guerre est employé : «Une nouvelle guerre», «Une guerre totale» (Le Figaro, 12 septembre); «État de guerre» (Le Point, 14 septembre), «Guerre contre l'Occident» (L'Express, 13 septembre), «Troisième guerre mondiale» (Le Monde, 12 septembre). Quelques remarques s'imposent. Ce concept de guerre fut également utilisé par certains dirigeants politiques, dont le président Bush, afin de qualifier du point de vue juridique

24. Ces éléments, loin d'être accessoires, conditionnent la lecture du récepteur. L'image «désintellectualise le message qu'elle contient. C'est aussi pourquoi elle donne beaucoup plus à reconnaitre qu'à connaitre». M. Mouillaud, 1989, Le journal quotidien, Lyon, Presses universitaires de Lyon, p. 82.

25. Sur cet aspect, C. Develotte, 2000, «Titre de presse et représentations d'autrui en temps de crise $»$, Mots, $\mathrm{n}^{\circ}$ 64, décembre, p. 23-38. 
les attentats. Un tel recours argumentatif relève de la distorsion. La guerre se définit dans la tradition du droit des gens et plus généralement dans toute la pensée politique depuis le $16^{\mathrm{e}}$ siècle comme un rapport d'État à État ${ }^{26}$. Avec le 11 septembre, se manifeste non pas un conflit classique entre entités étatiques, mais une action entreprise par un réseau de terroristes contre l'acteur le plus puissant de la planète. Recourir au concept de guerre est un moyen rationnel de combler une incertitude quant à la nature même de l'évènement mais qui ne répond pas vraiment à la qualification de celui-ci. Le mot d'apocalypse fait également l'objet de nombreuses occurrences : «L'apocalypse terroriste s'abat sur New York et Washington» (Le Figaro, 12 septembre); «Apocalypse au cœur de l'Amérique» (Libération, 12 septembre), «120 minutes d'apocalypse» (Le Nouvel Observateur, 14 septembre) ${ }^{27}$. L'utilisation de ce terme a pour principale conséquence de montrer le degré particulièrement élevé de la violence mais aussi d'inscrire l'évènement au cœur du mythologique et de l'eschatologique. En effet, la notion d'apocalypse trouve sa formulation dans le patrimoine biblique de l'Ancien et du Nouveau Testament ainsi que dans l'héritage musulman notamment salafiste. Les terroristes ont agi dans une perspective de réalisation apocalyptique que les médias occidentaux reprennent dans leur intelligibilité de l'évènement.

Cette intensité soulignée avec récurrence ouvre naturellement la voie à une interprétation des attentats en termes de rupture. L'un des critères qui permet de cristalliser un «vrai évènement» réside dans son inscription temporelle. S'il rime avec avènement d'une nouvelle ère ou si, selon les termes d'Hannah Arendt, à travers lui «le fil de la tradition est rompu» ${ }^{28}$, alors, il correspond à un véritable évènement qui invite à penser, à examiner la réalité sous un prisme inédit. Dans la presse écrite, la lecture du 11 septembre répond bien à cette particularité. L'ensemble des locuteurs souligne la nature historique des attentats, laquelle constitue non seulement la fin de l'après-guerre froide mais l'entrée dans le $21^{\mathrm{e}}$ siècle. Une telle lecture renvoie au souffle de l'évènement, sa «sidération»,

26. J. J. Rousseau : «[la guerre] n'est point une relation d'homme à homme mais une relation d'État à État, dans laquelle les particuliers ne sont ennemis qu'accidentellement, non point comme hommes ni même comme citoyens, mais comme soldats. [...] Chaque État ne peut avoir pour ennemis que d'autres États et non pas des hommes, attendu qu'entre choses de diverses natures on ne peut fixer aucun vrai rapport». Du contrat social, Paris, GarnierFlammaron, 1966, p. 47.

27. Le Nouvel Observateur utilise aussi d'autres termes qui participent du même champ lexical : «le chaos », «l'innommable». Le Monde du 12 septembre utilise l'expression "L'Amérique frappée, le monde saisi d'effroi».

28. H. Arendt, 1972, La Crise de la culture, Paris, Gallimard (Folio), p. 25. 
caractéristique récurrente dans le traitement journalistique selon la médiologie $^{29}$. À titre d'illustration, Libération publie plusieurs éditoriaux qui rendent compte de cette rupture stratégique dans l'histoire du système international. Yann Moix considère le 11 septembre comme «un point de départ d'une ère nouvelle de l'humanité ${ }^{30}$. Quant à Serge July, il écrit :

Cette offensive terroriste et sa réussite technique (meurtrière, médiatique et planétaire) constituent un évènement majeur, un changement stratégique fondamental, qui sanctionne le basculement dans un monde nouveau dominé par le nouveau désordre mondial. [...] Un Hiroshima terroriste sur une capitale, c'est désormais possible. Tel est le message du 11 septembre. ${ }^{31}$

Lévènement résonne comme une crise extraordinaire à la fois pour les valeurs occidentales mais aussi pour les États qui, jusqu'alors, pensaient échapper à l'insécurité depuis la fin de la Seconde Guerre mondiale. Le 11 septembre s'apparente à un coup de tonnerre puisqu'il remet en question le principe de sanctuarisation des territoires nationaux $\mathrm{du}$ «Nord». En temps de crise, souligne Julien Freund, la double nécessité de combler un vide de sens et de répondre à un contexte d'incertitude angoissante s'exprime avec force ${ }^{32}$. Les locuteurs médiatiques cherchent alors des ressources argumentatives afin de rendre intelligible la situation. L'imaginaire constitue l'une de ces ressources.

\section{Un imaginaire poreux}

Les attentats du 11 septembre génèrent nombre d'interprétations s'inscrivant dans le prolongement théorique du choc des civilisations. Celui-ci offre une grille de lecture simplifiée qui autorise une intelligibilité immédiate de l'évènement : la religion musulmane par essence violente explique la motivation première des terroristes, lesquels ont entrepris une attaque en bonne et due forme à l'encontre des valeurs occidentales. Une telle présentation schématique se manifeste sous la plume de John Vinocur (International Herald Tribune du 13 septembre 2001) ou bien de la journaliste italienne Oriana Fallaci qui, dans un article intitulé «Rage et orgueil» du Corriere della Sera, définit le 11 septembre comme l'avènement d'une nouvelle guerre des religions ${ }^{33}$.

29. Par exemple, F.B. Huyghes, 1999, «Croire contre», Cahiers de médiologie, ${ }^{\circ}{ }^{8}$, p. 12-13.

30. Libération, 14 septembre 2001, p. 29.

31. Libération, 13 septembre 2001, p. 48.

32. J. Freund, 1976, «Observations sur deux catégories de la dynamique polémogène, de la crise au conflit», Communications, ${ }^{\circ} 25$, p. 102.

33. Voir également son ouvrage La rage et l'orgueil, Paris, Plon, 2002. 
Cette référence au choc des civilisations est-elle tout aussi saillante au sein des quotidiens et hebdomadaires retenus dans l'analyse ? Si certaines plumes au sein du Figaro ou de L'Express diffusent parfois une explication culturaliste voire «huntingtonienne», l'ensemble des journaux appréhendé tend à prendre ses distances par rapport à l'argumentation de l'auteur américain; ce qui a pour principale conséquence de relativiser dans un premier temps, le caractère immuable de l'imaginaire occidental quant aux identités arabe et musulmane.

Deux journaux en particulier présentent un certain nombre d'analyses culturalistes en vue de rendre intelligible le 11 septembre. Dans Le Figaro, tout d'abord, l'éditorialiste Max Clos exprime à plusieurs reprises durant le mois de septembre 2001, une approche qui dénonce le caractère belliqueux d'origine essentialiste sous-jacent à la religion musulmane. La représentation des relations internationales qu'il véhicule repose sur une dichotomie irréductible entre les cultures occidentale et musulmane. Ainsi, le 14 septembre, il soutient :

Il s'agit bien d'une guerre. Et pas seulement contre les États-Unis. C'est tout l'Occident démocratique qui est menacé au nom d'une religion conquérante, acharnée à imposer un système de valeurs incompatibles avec les nôtres. Dans cette guerre, les Occidentaux ont le devoir moral et l'obligation pratique - s'ils veulent survivre de choisir leur camp : s'occuper du droit à la vie des hommes de notre civilisation. ${ }^{34}$

L'interprétation de Max Clos insiste sur la nécessité d'une réponse occidentale active dans une perspective de «survie». Il procède à un amalgame réducteur qui associe différents phénomènes sans s'interroger sur leurs origines politiques et sociales. Pour lui, la doctrine musulmane explique tous les comportements belliqueux manifestés par les musulmans de par le monde : «Comment ignorer que les criminels qui ont frappé le cœur des États-Unis, ceux qui égorgent en Afrique et qui oppriment les femmes en Afghanistan, le font au nom d'Allah ? $»^{35}$. Il réitère son point de vue le 28 septembre en simplifiant à l'extrême la motivation première des acteurs terroristes. Ce n'est que l'islam et exclusivement l'islam qui explique la mobilisation des terroristes :

Les menaces terroristes sont préparées au nom de l'Islam. Il serait imbécile de ne pas se préparer à combattre les tenants d'une religion qui proclame son intention de livrer une guerre sainte. Une guerre sainte a été déclarée à l'Occident. Nous sommes en danger, en situation de légitime défense et l'ennemi est identifié. Le choix est entre se battre ou se laisser égorger comme des moutons bêlants. ${ }^{36}$

34. Le Figaro, 14 septembre 2001, p. 20.

35. Ibid.

36. Le Figaro, 28 septembre 2001, p. 15. 
Un tel discours présente des distorsions assez fortes par rapport à la réalité. Max Clos affirme que non seulement l'ennemi est clairement défini (ce que les dirigeants occidentaux, américains y compris, ont quelque peu du mal à formuler depuis les attentats) mais aussi que la guerre sainte a été formellement et officiellement annoncée par «l'Islam». Le journaliste oublie que Oussama Ben Laden n'a absolument pas la compétence ni surtout l'autorité pour déclarer le djihad : les représentants de l'un des centres les plus influents de l'Islam (l'université d'al-Azhar au Caire) ont d'ailleurs fortement souligné ce fait et critiqué les appels à la violence de Ben Laden ${ }^{37}$.

Dans son numéro du 13 au 19 septembre, L'Express reprend également l'argumentation culturaliste et, par divers titres chocs comme «La guerre contre l'Occident», «La fracture entre civilisations», «La troisième guerre mondiale entre le terrorisme islamiste et l'Occident», s'inscrit totalement dans le sillage de Samuel Huntington ${ }^{38}$. Ainsi, la date du 11 septembre sonne comme «le premier jour de la guerre civilisationnelle entre l'Islam et l'Occident ${ }^{39}$. Le journal n'hésite pas à faire paraitre une carte mondiale des civilisations, comme l'avait fait en son temps l'auteur américain, mais également à insérer dans le numéro un entretien avec l'orientaliste Bernard Lewis connu pour sa conception étriquée du djihad et des rapports que l'Islam entretiendrait avec la violence $^{40}$. La guerre entre les deux civilisations s'expliquerait sur la base d'un sentiment d'infériorité éprouvé par les musulmans à partir de l'effritement de leur empire au Moyen Âge. Ainsi, «la perte progressive et irréversible de la prééminence de l'Orient, de l'Islam sur l'Occident taraude le monde musulman depuis le $13^{\mathrm{e}}$ siècle $»^{41}$. Là encore, le journal ne précise pas les conditions politiques et sociales qui peuvent éclaircir l'irruption d'un discours partisan à caractère violent, lequel s'inspire de la doctrine afin de justifier, de manière étriquée et critiquable, des actions terroristes. S'inscrivant dans le temps long, l'interprétation mobilisée réactive des préjugés occidentaux à l'encontre des altérités arabe et musulmane réifiées et unifiées. L'Express conclut par l'énonciation d'un clivage Nord-Sud qui succèderait à l'opposition Est-Ouest dans une perspective

37. B. Étienne, 2002, Les amants de l'apocalypse. Pour comprendre le 11 septembre, Paris, Éditions de l'aube, p. 21.

38. Cité à la page 40, il constitue une référence théorique qui parcourt implicitement l'ensemble du numéro.

39. L'Express, 13 au 19 septembre 2001, p. 42.

40. Voir notamment B. Lewis, 1990, «The roots of Muslim rage», Atlantic Monthly, septembre, p. 2. Pour une critique de cette lecture de l'Islam, voir J. Esposito, 1992, The Islamic Threat. Myth or Reality, Oxford, Oxford University Press, p. 178.

41. L'Express, 13 au 19 septembre 2001, p. 37. 
assez similaire aux différentes représentations médiatiques diffusées lors de la guerre du Golfe en $1991^{42}$.

Dans son numéro suivant, $L^{\prime}$ Express manifeste une certaine continuité dans l'interprétation avec des phrases du type «la guerre sainte ne s'arrêtera plus » ${ }^{43}$. Toutefois, une inflexion apparait dans le sens où le journal véhicule des représentations alternatives qui ont pour principale conséquence de corriger l'hypothèse d'une dichotomie civilisationnelle. Des éditoriaux ainsi que des entretiens avec des spécialistes ou bien des responsables diplomatiques expriment des critiques à l'encontre de la théorie du choc des civilisations. Ainsi, dans L'Express ${ }^{44}$, une chronique de Bernard Guetta souligne les faiblesses du manichéisme et critique l'emploi du terme «croisade» dans la bouche de certains hommes politiques comme le président Bush J.-R.

Il convient de noter le même type de phénomène dans Le Figaro. Parallèlement aux diatribes de Max Clos, le journal fait paraitre plusieurs entretiens qui remettent en question l'interprétation de Samuel Huntington : Thierry de Montbrial (18 septembre, p. 14), Joseph Macé-Scaron (19 septembre, p. 14), Aly Mayer El Sayed, ambassadeur d'Égypte en France (20 septembre, p. 15), Maxime Rodinson (28 septembre, p. 14). Qui plus est, dans un éditorial du 14 septembre, Pierre Rousselin s'inscrit en faux par rapport à une lecture culturaliste du 11 septembre :

Puisque Oussama Ben Laden est le suspect numéro un, il est nécessaire d'éviter à tout prix ce que Samuel Huntington appelle le choc des civilisations. L'ennemi reste le terrorisme, et notamment les réseaux islamiques radicaux. Le monde arabe et musulman doit se rallier à l'Occident. ${ }^{45}$

Plus généralement, les autres vecteurs médiatiques analysés adoptent une ligne de lecture clairement anti-culturaliste. Ils critiquent la portée explicative du choc des civilisations. Le Nouvel Observateur publie un éditorial de Jean Daniel au cours duquel différents arguments sont avancés dont la double nécessité de distinguer réveil religieux et intégrisme d'une part et de considérer le nombre majoritaire de victimes du terrorisme islamiste dans les États arabes et arabo-musulmans d'autre part ${ }^{46}$. Le Point publie deux pages de critique à l'égard de Samuel Huntington dans son numéro du 14 septembre ${ }^{47}$. Dans Libération, des entretiens avec Mike Rogin et François Burgat fustigent les interprétations

42. Ibid, p. 38.

43. L'Express, du 20 au 26 septembre 2001, p. 19.

44. Ibid.

45. Le Figaro, 14 septembre 2001, p. 10.

46. Le Nouvel Observateur, 20 au 26 septembre 2001, p. 58.

47. Le Point, 14 septembre, p. 42-43. 
en termes de guerre entre Orient et Occident ${ }^{48}$. Enfin, Le Monde souligne les travers d'une lecture conflictuelle de l'évènement entre le monde musulman et l'Occident ${ }^{49}$.

Ainsi, les représentations d'un Islam par essence belliqueux inscrites dans la mémoire occidentale resurgissent avec une certaine intensité dans l'après-11 septembre. Reflet d'un imaginaire toujours présent, elles présentent toutefois un caractère poreux dans le sens où elles ne sont pas constantes et qu'elles font face à d'autres interprétations de l'évènement dont les ressorts s'éloignent du dualisme culturel. Ces interprétations alternatives semblent progressivement s'imposer durant le mois de septembre 2001. Peut-on conclure, dès lors, à une fragilité de l'imaginaire occidental ? Les vecteurs médiatiques révèlent-ils l'effilochage définitif des représentations simplifiées de l'islam et de l'arabité en France?

\section{Une imaginaire tenace par occultation}

L'imaginaire occidental à l'encontre des altérités arabe et musulmane demeure actif mais sous une forme euphémisée. Il se donne à voir dans les interstices des commentaires et surtout par le biais d'occultations. En effet, les silences et le non-dit apportent un éclairage sur le traitement de l'information tout aussi déterminant que l'analyse de l'exprimé ${ }^{50}$. À cet égard, l'analyse du corpus révèle trois caractéristiques majeures.

Tout d'abord, la question des relations entre la religion musulmane et la violence - et plus particulièrement le djihad - ne fait pas l'objet d'une approche historique $^{51}$. Elle demeure cantonnée dans une perspective doctrinale, à savoir recenser les différents versets coraniques au sein desquels le concept de djihad est appréhendé. Conçu avant tout comme l'«effort» du croyant en vue de s'épanouir dans la religion, le djihad ne présente pas initialement de caractère belliqueux. Il renvoie à l'acception de "guerre sainte» sous l'effet de certaines conditions historiques bien précises, lesquelles autorisent une remise en cause de l'essentialisme guerrier de l'islam. En effet, les versets cités afin de justifier

48. Libération, 13 septembre 2001, p. 19.

49. Le Monde, 18 septembre, p. 16.

50. Sur la question des implicites linguistiques, C. Kerbrat-Orecchioni, 1986, L'Implicite, Paris, Armand Colin, p. 24.

51. Il s'agit là d'une spécificité inhérente à la presse mais plus généralement des représentations véhiculées en Europe. Réintroduire cette approche historique engendre une autre façon de considérer l'Islam comme le suggère Mohammed Arkoun, 1986, L'islam. Morale et politique, Paris, Desclée de Brouwer. 
le djihad correspondent au moment où Muhammad entend reconquérir La Mecque par la force. La violence qu'il manifeste sur le plan du discours n'a certainement pas pour cible les juifs ou les chrétiens (les gens du Livre) mais ceux qui refusent la révélation musulmane. En d'autres termes, les agitateurs koraïchites et polythéistes susceptibles de créer un désordre (fitna) au sein de la communauté musulmane ${ }^{52}$. Le Coran condamne sévèrement le suicide et considère la guerre sainte d'abord et avant tout par rapport aux enjeux internes de l'islam. Qui plus est, cette guerre doit être entreprise sur la base de certains principes et après le combat, l'équité, le respect et la miséricorde sont requis. La réduction de l'évènement à son caractère religieux s'inscrit dans une certaine constance du traitement de l'information puisque la guerre du Golfe en 1991 avait d'ores et déjà suscité cette focalisation du regard explicatif ${ }^{53}$. Elle traduit la persistance d'une perception occidentale accordant une préséance à la question de la doctrine musulmane depuis le Moyen Âge ${ }^{54}$.

En privilégiant la dimension théologique, la presse écrite occulte les motivations véritables des fanatiques qui se sont transformés en terroristes. Il s'agit là de la seconde absence majeure que manifeste le traitement de l'information à la suite du 11 septembre. La pulsion de mort qui les a animé ne résulte pas d'une eschatologie inscrite dans le texte coranique mais d'une vision fataliste du monde ici-bas dont le ressort vertueux peut faire l'objet d'une réhabilitation via la destruction. Les terroristes pensent ainsi étendre la Cité idéale céleste à toute l'humanité à partir du déclenchement de l'apocalypse. Ils agissent selon une conception monolithique de l'islam, laquelle ne représente en aucun cas sa richesse, sa pluralité et surtout son ouverture à l'Autre. Le terroriste fanatique profane l'altérité et considère la mort de celle-ci comme expiatoire. En définissant ce comportement de sacrilège comme inhérent à la religion musulmane, les perceptions occidentales alimentent le jeu des terroristes. Elles occultent la spécificité même du fanatisme commun d'ailleurs à toutes les ramifications des religions monothéistes ${ }^{55}$.

Dans le même ordre d'idées, les mécanismes politiques et sociaux qui favorisent l'essor du néo-fondamentalisme sont écartés par les vecteurs médiatiques étudiés. Le néo-fondamentalisme n'a finalement rien à voir avec la nature des

52. B. Étienne, ouvr. cité, p. 22-24.

53. Ainsi, John Esposito insiste sur l'impact du concept de la guerre sainte afin de saisir l'essence de l'évènement de 1991 : «La primauté des intérêts politiques et des questions politiques est écartée ou éclipsée par la vision d'une vieille rivalité entre eux et nous ». J. Esposito, ouvr. cité, p. 179.

54. Sur ce phénomène, D. Norman, 1960, Islam and the West, Édimbourg, The University Press, p. 269.

55. B. Étienne, ouvr. cité, p. 60 et suiv. 
mouvements islamistes du $20^{\mathrm{e}}$ siècle issus des années 1920 et réactivés sous l'effet de la révolution iranienne ${ }^{56}$ et, qui plus est, il déroge aux règles coraniques. Il prend racine sur l'échec des projets d'islamisation dans les sociétés arabes et musulmanes. Tous les déçus de la modernité (jeunes diplômés, frustrés de l'arabisation, etc.) dans ces sociétés mais aussi dans les démocraties occidentales cherchent de nouvelles ressources de mobilisation et d'action. C'est ce rapport conflictuel à la modernité tant sur le plan des valeurs que sur le plan de sa réalisation politique (critique des gouvernements qui n'ont pas su associer la population lettrée et formée au fonctionnement de l'administration et de la société) qui constitue le facteur principal expliquant l'engouement de certains individus pour des mouvements terroristes comme al-Qaida.

Enfin, le troisième oubli renvoie à un mécanisme de refoulement. Les quotidiens et hebdomadaires analysés rejettent le terreau sémitique commun à toutes les religions monothéistes. L'islam ne constitue pas une altérité directe de la chrétienté en particulier, ou de l'Occident en général. La religion musulmane s'inscrit au contraire dans l'héritage «gréco-biblique» ainsi que dans l'histoire du bassin méditerranéen ${ }^{57}$. Ces sources partagées entre les civilisations «du Livre» ne sont pas mentionnées ni soulignées.

À la suite des évènements de la guerre du Golfe, l'imaginaire occidental à l'égard de l'islam et de la culture arabe fit l'objet d'une réactivation. Les attentats du 11 septembre tendent à révéler des mécanismes similaires mais avec des spécificités. Si le traitement de l'information traduit parfois la présence du choc des civilisations lié à une représentation étriquée de l'islam, il présente toutefois un imaginaire plutôt poreux et actif sous forme de latence, c'est-à-dire par occultation de certains phénomènes explicatifs de l'évènement. Ainsi, la façon dont la presse écrite envisage l'altérité arabo-musulmane demeure hermétique aux origines psychologiques, sociales et politiques de ce dernier. Dans Les nourritures terrestres, Gide écrit : «que l'importance soit dans ton regard, non dans la chose regardée». Après le 11 septembre, le regard de certains vecteurs médiatiques semble encore parcellaire.

56. Pour une distinction entre ces deux mouvances, O. Roy, 2002, L'islam mondialisé, Paris, Seuil. Voir également, O. Roy, 2001, «Ben Laden et ses frères», Politique internationale, $\mathrm{n}^{\circ}$ 93, automne, p. 67 et suiv.

57. P. Chaunu, 1991, «Les Arabes font aussi partie de l'Occident», Hérodote, ${ }^{\circ}$ 60-61, p. 41. 